\title{
PROMISING ELECTROMECHANICAL AXIAL CONVERTERS
}

\author{
Bagaudin Khamidovich Gaitov \\ Department of Electrical Engineering and Electrical \\ Machines \\ Kuban State Technological University \\ Krasnodar, Russia \\ e-mail: jlms@mail.ru
}

\author{
Alexander Valeryevich Samorodov \\ Department of Electrical Engineering and Electrical \\ Machines \\ Kuban State Technological University \\ Krasnodar, Russia \\ e-mail: Alex.Samorodoff@gmail.com
}

\author{
Lev Efimovich Kopelevich \\ Department of Electrical Engineering and Electrical \\ Machines \\ Kuban State Technological University \\ Krasnodar, Russia \\ e-mail: kkllev1@mail.ru
}

\author{
Alexey Alexandrovich Sharshak \\ Department of Electrical Engineering and Electrical \\ Machines \\ Kuban State Technological University \\ Krasnodar, Russia \\ e-mail: vip.sharshak@mail.ru
}

Annotation-The work presents original designs of axial electromechanical energy converters for application in various fields: oil industry, renewable energy generation, welding equipment and electric power supply.

Keywords-electromechanical energy converter, axial design, stator, rotor, mass-dimensional characteristics, magnetic conductor, renewable energy sources.

\section{INTRODUCTION}

Over a number of years, the Department of Electrical Engineering and Electrical Machines of Kuban State Technological University have been developing electromechanical axial converters of various design.

Axial converters have both strong points and drawbacks. We believe, the key advantages are ability to create aggregated designs with several magnetic conductors and simple implementation of direct drive. They are called driving machines. In addition, the authors contributed to the development of practically zero-waste technology for axial magnetic conductor production. A plenty of technical problems, that are challenging for conventional electromechanical energy converters, can be solved using axial machines.

The present article discovers several designs of axial electromechanical energy converters. All designs and technical solutions are patented in the RF.

\section{RESULT AND DISCUSSION}

Multi-phase axial induction regulator.

The multi-phase axial induction regulator (Fig. 1) consists of fixed toroid magnetic conductor 1 with primary multi-phase (for instance, twelve-phase) winding 2, fixed toroid magnetic conductor 3 with secondary three-phase winding 4 , the starts of corresponding phases of which are electrically coupled with the starts of primary multi-phase winding 2 via three-phase electromechanical switch 5 with sliding contacts 6 (Fig. 2) and case 7. Three-phase electromechanical switch 5 with sliding contacts 6 in its turn ensures the coupling of a group of phases of primary multi-phase winding 2 with corresponding phases of the three-phase supply mains and with corresponding phases of the secondary three-phase winding.

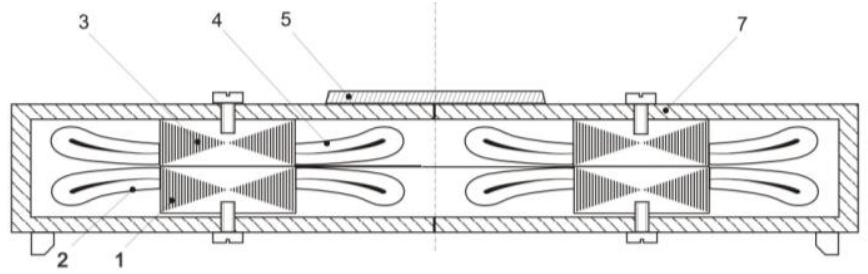

Fig. 1. Multi-phase axial induction regulator (general view)

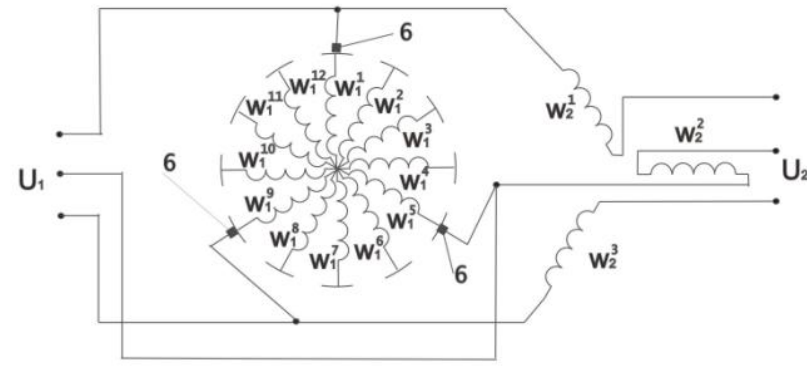

Fig. 2. Multi-phase axial induction regulator (electric diagram)

Multi-phase axial induction regulator enables discrete voltage regulation. The required regulation accuracy is determined by the number of phases $m$ of the primary multiphase winding. The proposed design allows appreciably simplifying the prototype regulator where the complexity is conditioned by necessary reciprocal displacement of its 
magnetic conductors and, hence, necessary implementation of highly stressed bearing joints. It also allows increasing the power factor and efficiency factor of the regulator, which low values for the prototype regulator are caused by the air gap between the magnetic conductors necessary for their reciprocal displacement, which, in its turn leads to increased magnetic resistance, and, hence, increased energy losses.

\section{Multi-phase transformer.}

Fig. 3 gives the general view of suggested multi-phase transformer (in current configuration, 12-phase transformer with three-phase secondary winding). Fig. 4 depicts the electrical scheme.

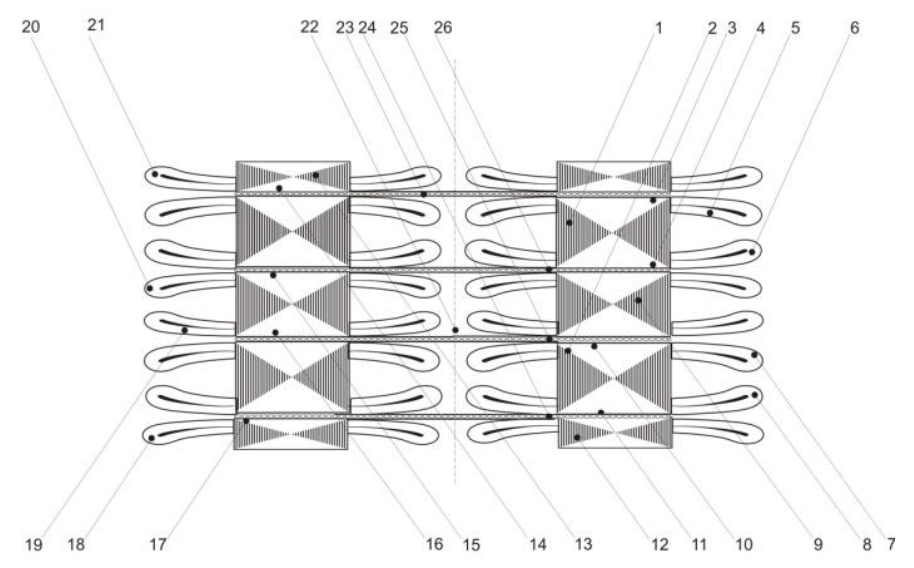

Fig. 3. Multi-phase transformer (general view)

Multi-phase (in given configurations, 12-phase and 36phase) transformer consists of internal axial magnetic conductors (1 and 2 in Fig. 3), each having two active end faces (3, 4, 10 and 11 in Fig. 3) with slots for primary threephase windings $(5,6,7$ and 8 in Fig. 3), internal axial magnetic conductors 9 , each having two active end faces (15 and 16 in Fig. 3) with slots for secondary multi-phase (in given configurations, three-phase) windings (19 and 20) and two side axial magnetic conductors (12 and 13) each having one active end face (14 and 17) with slots for secondary multiphase windings (18 and 21). Internal axial magnetic conductors 9 with slots with laid in secondary multi-phase windings (19 and 20) are displaced relative to internal axial magnetic conductors (1 and 2) with slots having primary three-phase windings (5, 6, 7 and 8). Such design allows changing the number of supply voltage phases virtually in any range.

Multi-phase aggregated welding transformer.

The work suggests a multi-phase aggregated welding transformer which improves direct-current welding quality due to the high quality of rectified voltage.

Fig. 5 illustrates the general sectional view of the suggested multi-phase aggregated welding transformer.

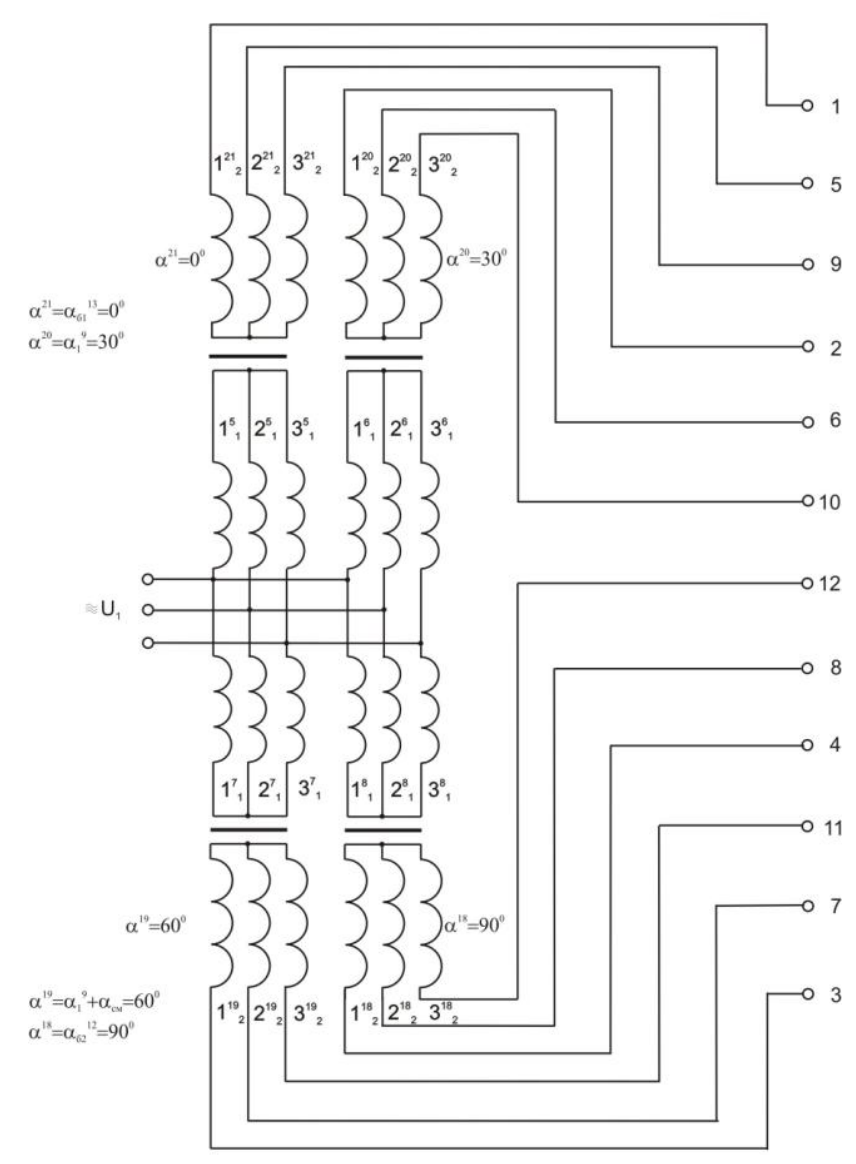

Fig. 4. Multi-phase transformer (electric diagram)

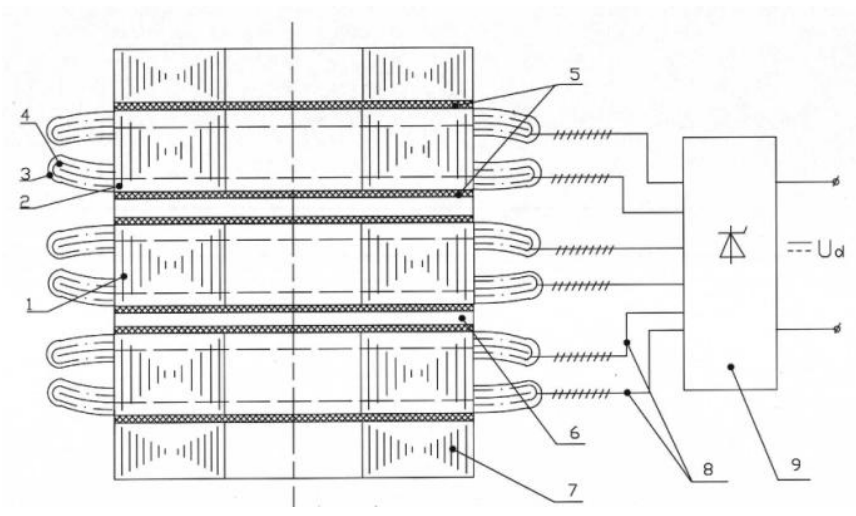

Fig. 5. Multi-phase aggregated welding transformer

The multi-phase aggregated welding transformer (Fig. 5) consists of several internal axial magnetic conductors 1 , each having two end faces with slots 2 for primary three-phase winding 3 (solid lines) and secondary multi-phase (e.g., ninephase) winding 4 (dash-dotted lines), two side no-slot axial magnetic conductors 7 adjoining internal magnetic conductors 1 by end faces through non-magnetic gaskets 5 . The internal magnetic conductors adjoin each other by active end faces through non-magnetic gaskets 5 separated by single 
intermediate yoke 6 made from electrical steel sheet. The ends of primary three-phase windings 3 are coupled with threephase mains 8 , while the ends of secondary multi-phase (e.g., nine-phase) windings 4 are coupled with multi-phase (e.g., nine-phase) full-wave rectifier 9 providing high quality of welding due to decreased ripples of rectified voltage. The number of phases of primary 3 and secondary 4 windings in Fig. 5 is correspondingly illustrated by three and nine transverse inclined strokes.

Thus, the quality of DC voltage rectified from $\mathrm{AC}$ is significantly higher with increased phase of rectified voltage (Fig. 6). In this connection, the multi-phase aggregated welding transformers can be used without ripple filters.

\section{Centrifugal pumps.}

The authors have developed a series of designs of combined axial centrifugal pumps for various industrial application [1]. The developed designs have enhanced reliability and better energy characteristics versus existing axial pumps.

The developed designs are protected by a number of RF patents.
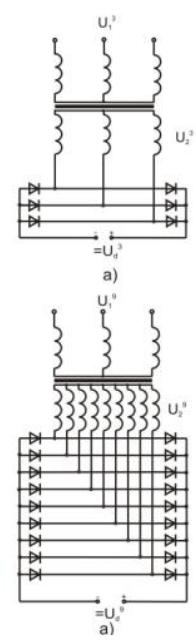

Fig. 6. Multi-phase aggregated welding transformer (operation principle).

\section{Oil separators.}

The work proposes a design of combined axial-radial separator for oil processing with enhanced energy characteristics (Fig. 7).

The separator consists of electric motor (cylindrical part 21, axial part 2-2) with winding 3, two stator parts which front part is surrounded by pipes 4 filled with compound 5, separator drum 6 that simultaneously plays the role of the electric motor rotor, which is rigidly connected with axle 7 that is mounted in bearing assemblies 8 and 9. Separator drum 6 consists of base 10 with central pipe, separation disks 11 , cap 12, disk support 13 and clamping ring 14. Connection pipe 16 connects oil heater 15 with the inlet of pipes 4 , while connection pipe 17 connects outlet of pipes 4 with the internal part of separator drum 6 .

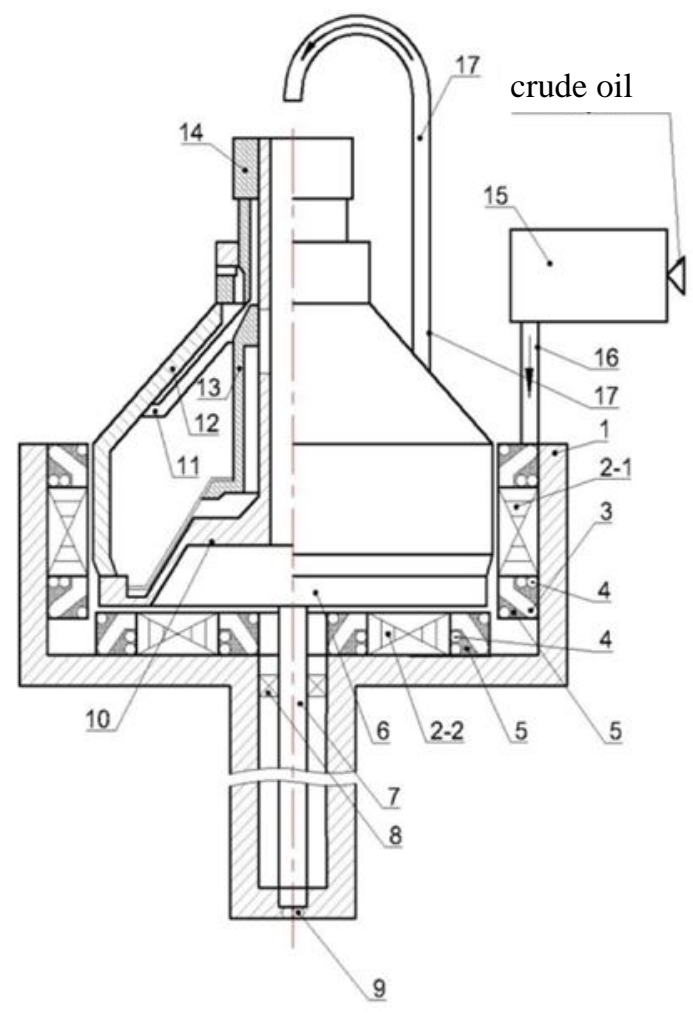

Fig. 7. Oil separator.

The setup enables direct drive of the separator drum without intermediate elements and recycling of the heat liberating in the windings and magnetic conductor of stators to warm up the separated oil.

\section{Renewable energy.}

Currently, an appreciable attention in the world is paid to green renewable energy [2-10].

The analysis of global trends in this field shows that the majority of works is aimed at the improvement of existing designs, development of new algorithms for controlling nonconventional energy generation systems, etc.

Novel electromechanical energy converters for power supply systems with renewable energy sources are practically developed by very few specialists.

To convert wind and solar energy into electric energy with their simultaneous combination, a number of original designs of axial generators were developed [11].

One of them is axial two-input contact-less electrical generator machine. The general view of the machine is in Fig. 2.

Such generator machine can combine and convert kinetic wind energy and solar energy generated by photovoltaics into DC electric energy and AC three- (or more) phase electric energy with more stable output parameters as compared to conventional electromechanical energy converters. 
Axial two-input contact-less electric generator machine consists of case 1, constant multi-polar magnet 2 of subexciter inductor, side axial magnetic conductor 3 with multi-phase winding 4 of subexciter armature, single-phase winding 5 of exciter excitation and auxiliary winding 6 of exciter excitation that is connected to a DC source through contacts 19 (Fig. 8), internal axial magnetic conductor 7 with multi-phase winding 8 of exciter armature and single-phase winding 9 of the main generator excitation, side axial magnetic conductor 10 with multi-phase (in Fig. 2, nine-phase) winding 11 of the main generator armature, axle 12 mounted in bearing assemblies 13 and 14 and rigidly connected with constant multi-polar magnet 2 of subexciter inductor through disk 15 and with internal axial magnetic conductor 7 through disk 16.

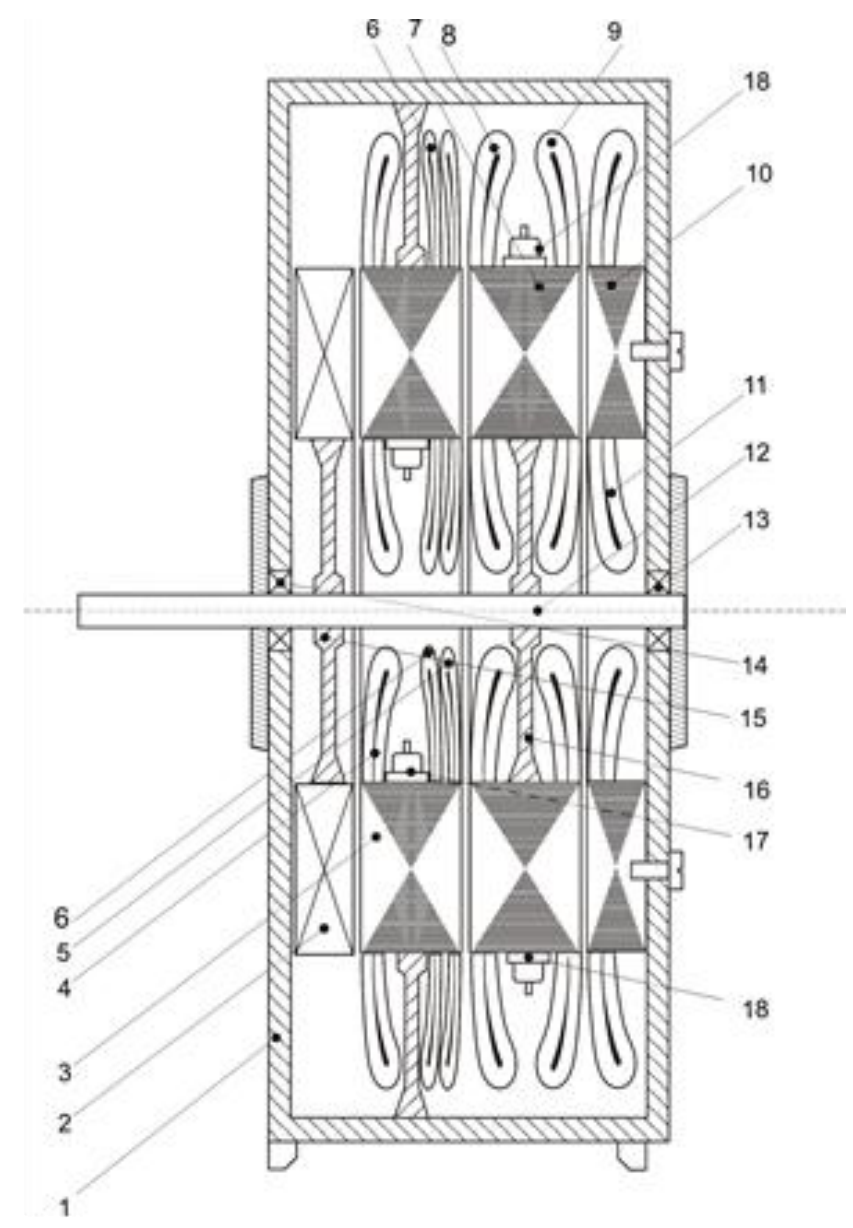

Fig. 8. Axial two-input contact-less electrical generator machine

The rotor is formed by the elements of subexciter magnetic systems, exciter and main generator 9 (constant axial multipolar magnet 3 of subexciter inductor and axial rotating magnetic conductor 7 with multi-phase winding 8 of exciter armature and single-phase winding 9 of main generator excitation) rigidly fixed of the axle using the disks. The rotor axle is mounted in bearing assemblies 13 .

The rotor rotates relative to the elements of the magnetic systems of the subexciter, exciter and main generator (side axial magnetic conductor with multi-phase main generator armature winding, internal axial magnetic conductor with multi-phase subexciter armature winding, main and auxiliary single-phase exciter excitation windings) mounted on the case.

In addition, other designs of axial generators for renewable energy sources were developed.

All developed axial generators are protected by RF patents.

Moreover, the creation of promising designs of electromechanical energy converters for renewable energy generation and distribution is supported by the Russian Foundation for Basic Research and the Administration of Krasnodar Krai.

The investigation was financially supported by the Russian Foundation for Basic Research and Administration of Krasnodar Krai within scientific project no. 16-48-230500 r_a.

\section{References}

[1] B.Kh. Gaitov, Ya.M. Kashin, L.E. Kopelevich, A.V. Samorodov, V.A. Kim, "Setup for oil separation," Oil industry, Vol. 7, pp. 90-92, 2017.

[2] R.A. Amerkhanov, A.V. Bogdan,V.A. Butuzov, "Horizons of Krasnodar Krai power generation development using renewable energy sources," Energy conservation and water treatment, Vol. 3, pp. 52-55, 2005.

[3] P.P. Bezrukikh, "State and horizons of renewable power generation development," Electric engineering, Vol. 9, pp. 3-10, 2008.

[4] G.V. Stepanchuk, K.S. Morenko, "Usage of low-quality electric energy of a wind power station with two-rotor generator," Innovations in agriculture, Vol. 1 (3), pp. 63-65, 2013.

[5] O.I. Shutkin, Ecological and economical assessment of the competitiveness of solar energy generation projects in the Russian Federation: Cand. Thesis, Moscow, 2014.

[6] Frankfurt School-UNEP Centre/Bloomberg New Energy Finance (2015), Global Trends in Renewable Energy Investment, 2015.

[7] X.A. Ran, Z. Jiang, H. Xu, "Framework for uncertainty quantification and economic dispatch model with wind-solar energy," International Journal of Electrical Power and Energy Systems, Vol. 73, pp. 23-33, 2015.

[8] M. Sharafi, T.Y. ElMekkawy, E.L. Bibeau, "Optimal design of hybrid renewable energy systems in buildings with low to high renewable energy ratio," RenewableEnergy, Vol. 83, pp. 1026-1042, 2015.

[9] The European wind energy association. Wind in power: 2009 European statistics, February 2012.

[10] Renewables 2015 Global Status Report, REN21 2015. URL: http://www.ren21.net/status-ofrenewables/global-status-report/.

[11] B.Kh. Gaitov, Ya.M. Kashin, A.Ya. Kashin, L.E. Kopelevich, A.V. Samorodov, Axial two-input contact-less wind and solar generator, Patent 2561504 Russian Federation, Applicant and patent owner KubSTU, June 16, 2014. 